\title{
Enfermedades transmitidas por los alimentos: Análisis del riesgo microbiológico
}

\author{
Lucia J. González, Fernanda N. Martínez, Laura Rossi, Mariela Tornese y Alcides Troncoso
}

\section{Risk analysis of foodborne diseases: Microbial risk assessment}

The basic goals of risk assessment include the following: to identify potentially hazardous situations and apply appropriate methods to estimate the likelihood that a hazard occurs. In The uncertainty in that estimate, to provide alternative solutions to reduce the risk, estimate the effectiveness of those solutions, provide information to base a risk management decision, and estimate the uncertainty associated with the analysis. Risk analysis provides the rational framework for assembling and then analysing the evidence relating to risk and presenting the results in a form that is easy to understand and then act upon fairly and effectively. Progress made is no excuse for arrogance. The present review is definitely not designed as the last word on risk analysis for foodborne diseases. Rather, this review has been designed to continue an evolving and necessary process and to provide a reference point that indicates the state of development in 2010.

Key words: Quantitative risk assessment, growth models, microbial risk.

Palabras clave: Evaluación cuantitativa de riesgos, modelos de crecimiento, riesgos microbianos.

\section{Introducción}

E 1 análisis de riesgo microbiano (ARM) es un proceso utilizado para evaluar los peligros ocultos en los alimentos, la probabilidad de exposición a estos y su impacto sobre la salud pública.

El ARM se realiza en cuatro fases:

- Identificación del peligro

- Caracterización del peligro

- Determinación de la exposición

- Análisis del riesgo

Los modelos de predicciones generados por el ARM se expresan como la probabilidad de adquirir una enfermedad de origen alimentario (ETA) y/o el número de muertes atribuidas a una ETA en una población, en un período dado. El ARM se esta usando cada vez más para la toma de decisiones que apuntan al manejo de los riesgos de patógenos de origen alimentario y como un método estándar para regular el comercio internacional de alimentos ${ }^{1}$.

\section{Nacimiento del análisis de riesgo}

Durante los últimos años de la década del ‘60 y los primeros de la década del '70 varios compuestos químicos incluyendo poluciones ambientales fueron identificados como cancerígenos. Esto provocó un cambio sustantivo en los procesos institucionales para evaluar la información de los riesgos para la salud y la manera en la cual los resultados eran traducidos en políticas regulatorias. Luego, hacia los primeros años de la década de los '80 una directiva del Congreso de E.U.A. establecía, darle nacimiento a la formación del Consejo Nacional de Investigación. El objetivo mayor del Comité fue definir cómo reforzar la confiabilidad y objetividad de las determinaciones científicas que forman la base de las regulaciones aplicables a cancerígenos y otros peligros para la salud publica. El resultado del trabajo del Comité fue sintetizado y publicado en 1983. Este reporte tuvo aceptación general por la comunidad científica y factibilidad real, dado que podía ser usado de muchas maneras para marcar el principio enunciado en el $\mathrm{ARM}^{2}$.

En los años recientes se han adoptado varios procesos de ARM para evaluar los riesgos de exposición a una ETA. Más específicamente en 1994, el Acuerdo General de Tarifas y Comercio estableció el Acuerdo en la Aplicación de Medidas Fitosanitarias y el Acuerdo a Barreras Técnicas para el Comercio. Estos dos acuerdos intentaron asegurar: que ningún Estado miembro esté impedido de adoptar e implementar medidas para proteger la salud a los humanos, animales y plantas y facilitar el libre comercio internacional de alimentos asegurando la protección de la salud pública. Es un hecho, el establecimiento de líneas de trabajo y de procedimientos por los cuales la conducta de los países miembros y de sus determinaciones de riesgo para garantizar la Seguridad
Universidad de Buenos Aires, Argentina.

Cátedra de Microbiología y Parasitología (ப, FNM, LR, MT, AT) Cátedra de Enfermedades Infecciosas (AT).

Recibido: 20 de mayo de 2010. Aceptado: 24 de junio de 2010

Conflicto de intereses: ninguno.

Correspondencia a: Alcides Troncoso microbiologiayparasitologia@ yahoo.com.ar 
Alimentaria están bajo los auspicios de la Organización Mundial de la Salud (OMS), Organización de Agricultura y Alimentos (FAO), Programa de Alimentos Standard, Comisión Codex Alimentarius (Codex). Hoy en día las determinaciones de ARM son un componente estándar, en el esfuerzo de proteger la salud publica y facilitar el libre comercio ${ }^{3}$.

\section{Clasificación de análisis de riesgo microbiano según proceso/resultado}

El ARM comprende dos categorías:

- Cualitativa

- Cuantitativa

En la primera, el riesgo es descrito como la probabilidad de enfermedad (alta versus baja) mientras que en la última es expresada como el número predecible de casos de enfermedad. En la Evaluación Cuantitativa del Riesgo Microbiano (ECRM) se procede a la estimación de los principales parámetros de crecimiento microbiano (fase de latencia, tasa máxima de crecimiento y densidad máxima de población), así como la probabilidad de crecimiento bajo diferentes condiciones ambientales sobre medios de cultivo. Una forma de ejemplificar cómo se monitoriza la calidad microbiológica y sensorial es el caso de los espárragos blancos, pasteurizados a diferentes temperaturas de almacenamiento, junto con la incidencia y evolución de crecimiento bacteriano. A través de la información obtenida, y junto con una recopilación de datos procedentes de la literatura científica, se aborda la aplicación de los modelos predictores desarrollados en un esquema de ECRM mediante la estimación del número final de células presentes en el momento de consumo y el número de casos/año sobre una población. La metodología constituye una vía relativamente sencilla de evaluar la seguridad microbiológica de un proceso alimentario mediante la integración de modelos matemáticos dentro de un esquema de $\mathrm{ARM}^{4}$.

La ECRM provee expresiones numéricas de riesgo, usa modelos de probabilidad para evaluar la posibilidad de efectos adversos en la salud humana debido a la exposición a microorganismos patógenos. Las predicciones son presentadas como distribuciones, más que como estimaciones puntuales. Este acercamiento ofrece varias ventajas, unas de las cuales es que la variabilidad de un factor contribuyente puede ser significativa, lo cual puede ser difícil de asignar apropiadamente por puntos de estimaciones solamente. Otra ventaja es que el ECRM facilita la identificación de los factores más importantes determinando la magnitud de un riesgo. Por último, los modelos cuantitativos son flexibles; los datos de entrada y los componentes de los modelos pueden cambiar rápi- damente mientras nueva información se torna accesible. Casi todos los ECRM hoy en día se consiguen usando el muestreo de Monte Carlo. Este es un muestreo aleatorio de probabilidad de distribuciones individuales para producir cientos o miles de escenarios de resultados ${ }^{5}$.

\section{Un enfoque general del análisis de riesgo microbiano}

La evaluación del ECRM en seguridad alimentaria es un componente del proceso de análisis del riesgo, seguido por su manejo y comunicación. La misma facilita el enfoque necesario para prever la importancia de la salud pública en la producción y prácticas de procesamiento de nuevos alimentos, la emergencia de patógenos trasmitidos por ellos, así como identificar los puntos críticos dentro del sistema de suministro de alimentos en el cual las intervenciones adicionales van a tener el mejor impacto para lograr la inocuidad de los alimentos y por lo tanto, en la reducción de peligros para la salud. Con la adopción de modelos probados que concuerden con un escenario mundial real, se asume que el uso de esta evaluación del ECRM en la industria alimenticia se convertirá en la principal herramienta para la evaluación de los riesgos ${ }^{3}$.

\section{Análisis de riesgo: Visión panorámica}

El ARM típicamente necesita acceso a un amplio rango de experiencia profesional e información. Esto incluye no sólo identificación del tipo, extensión, calidad y accesibilidad de la información, sino también organizaciones claves con responsabilidad y capacidad de decisión frente a los resultados del ARM. Para lograrlo se requiere de expertos en diversas disciplinas científicas incluyendo microbiología, química, epidemiología, medicina, matemática, estadística, toxicología y ciencias de los alimentos, que han probado ser necesarios para conducir ARM útiles. En consecuencia, es críticamente importante antes de adoptar una ECRM, contar con personal apropiadamente entrenado. A causa de su carácter interdisciplinario, usar un equipo de individuos con diferentes tipos de experiencia es una aproximación muy efectiva para conducir el análisis de riesgo. Asimismo, a causa de su conexión a varios niveles organizacionales (personal técnico/ científico para hacer el trabajo técnico, personal de comunicaciones para hacer la comunicación de riesgo, y directivos para tomar decisiones), es crítico para los análisis de riesgo, hacer un esfuerzo de equipo ${ }^{5}$.

El análisis de riesgo se constituye de tres etapas:

- Fase de planificación

- Fase de determinación (análisis del riesgo-ECRM)

- Fase de gerenciamiento, a su vez con dos componentes: 
- Estructura administrativa de las decisiones de riesgo

- Estrategia para la comunicación de riesgo

\section{Fase de planificación}

La formulación del problema, o la fase de planificación, es crítica para conseguir el resultado de la más alta calidad del análisis de riesgo. La planificación no debería excluir a ningún miembro del equipo para asegurarse que todos tengan participación en la formulación de las preguntas que la investigación responderá, y qué grado de incertidumbre en estas respuestas es aceptable. La fase de planificación debería incluir la discusión de criterios para la calidad del análisis, el cual deberá conducir las necesidades de la calidad de la información para el proyecto. Por ejemplo, si una sola estimación aproximada es necesaria y un alto grado de incertidumbre es aceptable, la calidad de la información de entrada puede no ser tan crítica como cuando un alto grado de certeza es necesario, en cuyo caso la calidad de la información y la calidad de todos los modelos usados necesitan ser desarrollados para asegurar un alto grado de certeza. La fase de planificación debe también coordinar las necesidades de los gerentes de riesgo (definiendo la cuestión que necesita respuesta) con la habilidad de los analistas (tomando en cuenta los recursos accesibles incluyendo experiencia personal, tiempo y modelos). Los comunicadores de riesgo deben estar también involucrados en la planificación para asegurar que la estrategia de la comunicación sobre estos riesgos sea tomada en cuenta desde el principio. Esta aproximación guía hacia una eficiente y efectiva estrategia para tomar decisiones sobre el riesgo ${ }^{6}$.

\section{Fase de determinación (análisis)}

\section{Proceso de ARM}

El análisis de riesgo es el proceso que permite la estimación de la probabilidad y severidad de un resultado en particular, dado un escenario bien definido. Esta medida de probabilidad y severidad es llamada riesgo estimado. El análisis de riesgo es el umbral de análisis en el cual la información esta investigada y asignada, los modelos son elegidos o desarrollados, y los resultados alcanzan un alto grado de confianza en las estimaciones. Nuevamente esto debería comparar las necesidades del análisis de riesgo, tal como están definidos en el escenario planeado?.

El ARM incluye cuatro puntos:

- Identificación del peligro (la identificación de agentes biológicos, químicos y físicos capaces de causar efectos adversos a la salud y que pueden estar presentes en un grupo de alimentos particular).

- Caracterización dosis/respuesta del peligro (la evaluación cualitativa y/o cuantitativa de la naturaleza de los efectos adversos a la salud asociada con el peligro).
- Determinación de exposición (evaluación cualitativa y/o cuantitativa de la probabilidad de ingesta de agentes biológicos, químicos y físicos vía alimentaria tanto como exposición de otras fuentes si fueran relevantes).

- Caracterización de riesgo (la integración de la identificación del peligro): caracterización del peligro y determinación de la evaluación de la exposición para proveer estimaciones cualitativas o cuantitativas de la probabilidad y gravedad de efectos adversos que pueden ocurrir en una población dada.

\section{Identificación del peligro}

La identificación del peligro es predominantemente un proceso cualitativo que consiste en detectar los microorganismos o sus toxinas, por ejemplo aquellas en las cuales la asociación entre la enfermedad y la presencia de un patógeno en un alimento, sea reconocida y confirmada como causa de una ETA. La información sobre los peligros puede obtenerse de la literatura científica, varias bases de datos, y a través de la convocatoria de expertos. La información pertinente puede ser encontrada de investigación básica y estudios clínicos, epidemiológicos y de vigilancia ${ }^{8}$.

\section{Caracterización del peligro}

El componente primario de la caracterización del peligro es la relación dosis/respuesta, definida como el análisis de la relación entre la magnitud de la exposición a un agente biológico, químico o físico y la gravedad y/o frecuencia de la asociación a efectos adversos para la salud. La caracterización del peligro de esta manera provee una descripción cualitativa o cuantitativa de la gravedad y duración de efectos adversos que pueden resultar de la ingestión de un microorganismo presente en los alimentos o sus toxinas. La relación dosis/respuesta puede ser derivada usando datos de seguimiento de investigaciones epidemiológicas. Por ejemplo, una combinación brindada por la epidemiología y la información derivada del estudio de los alimentos permite estimar la relación dosis/respuesta para Listeria monocytogenes, asociada al consumo de quesos contaminados. La extrapolación de estudios animales para determinar la dosis/respuesta puede ser también usada para analizar dosis/respuesta, tal como en el análisis del riesgo para L. monocytogenes ${ }^{5}$.

Varios factores importantes relacionados a los microorganismos deben ser tenidos en cuenta cuando se realizan caracterizaciones del peligro. Esto incluye los hechos que:

- los microorganismos pueden replicarse en los alimentos,

- la virulencia e infecciosidad del microorganismo pueden cambiar dependiendo de su interacción con sus hospederos y el medio ambiente,

- el material genético puede transferirse entre microorganismos llevando a la transmisión de resistencia a antimicrobianos y factores de virulencia, 
- los microorganismos pueden ser diseminados en forma interhumana a través de transmisiones secundarias y terciarias,

- los microorganismos pueden persistir crónicamente en ciertos individuos asintomáticos (portadores) llevando a la diseminación continua y posterior chance de expansión de la infección, y

- aunque aumente la probabilidad de adquirir una enfermedad con un número creciente de microorganismos patógenos consumidos, hay un potencial para que muy bajos niveles de bacterias puedan causar enfermedad como es el caso de Escherichia coli enterohemorrágica ${ }^{9}$.

De similar modo, los aspectos concernientes a la ecología microbiológica de alimentos deben ser cuidadosamente analizados, previo a la caracterización del peligro. Por ejemplo, los atributos de la comida tales como un alto contenido en grasa y sal (y efectos de la matriz alimentaria, en general) son importantes consideraciones para evaluar el potencial de la virulencia microbiológica. Como por ejemplo, estudios con Vibrio cholerae indican que la matriz alimentaria, tal como arroz cocido, provee capacidad buffer y puede tener impacto sustancial en la relación dosis/respuesta. Otras investigaciones han demostrado que un elevado tenor graso en la leche inhibe la colonización intestinal por Listeria sp pero no por Salmonella sp en ratas. Por último, es importante en este estudio de la caracterización del peligro, recordar que la virulencia microbiológica puede también estar afectada por muchos factores del hospedero tales como: incrementada susceptibilidad del hospedero a causa de una anomalía de las barreras fisiológicas, susceptibilidad particular del hospedero tales como edad, estado inmune, etc. y características de la población, tales como acceso al cuidado medico ${ }^{10}$.

\section{Determinación de la exposición}

En esta etapa se estima el nivel de patógenos o sus toxinas, y la probabilidad de su presencia en un alimento al tiempo de su consumo. Esto incluye una evaluación real y anticipada de la exposición humana al peligro. Los niveles de microorganismos pueden ser dinámicos $\mathrm{y}$, mientras ellos puedan ser mantenidos bajos, adhiriendo a controles de tiempo/temperatura adecuados durante el procesamiento de alimentos, pueden incrementarse sustancialmente con incorrecta conservación y/o manipulación de los alimentos o cuando estos son dejados a temperatura ambiente o en medio de anaerobiosis (botulismo de los alimentos) ${ }^{2}$.

La evaluación a la exposición, por lo tanto, usualmente describe el sendero desde la producción al consumo. Los escenarios pueden ser construidos para predecir el rango de posibles exposiciones. Cualitativamente los alimentos pueden ser categorizados de acuerdo a la probabilidad de que puedan estar contaminados desde su fuente de procedencia; si son capaces de permitir el crecimiento bacteriano a condiciones de temperatura y $\mathrm{pH}$ requeridas, si fueron sometidos a una sustancial manipulación abusiva, si fueron sometidos a procesos de recalentamiento inadecuado o algún otro paso crítico ${ }^{8}$.

A su vez, la presencia, crecimiento, sobrevivencia y muerte de patógenos microbianos en los alimentos son influidos por varios factores. Estos pueden incluir

- las características del microorganismo patógeno,

- la ecología microbiana de los alimentos,

- la contaminación bacteriana inicial de la materia prima de los alimentos,

- el nivel de sanitización, los procesos de control y los métodos de procesamiento,

- envasado, distribución y almacenamiento de los alimentos, y

- los pasos de la preparación de las comidas tales como la cocción y el manejo de la temperatura.

El potencial impacto entre el abuso del tiempo de exposición a temperaturas altas debería ser considerado para evaluar el grado de exposición a una bacteria peligrosa. Por ejemplo, los niveles de exposición humana a patógenos de origen alimentario pueden rápidamente incrementarse por un millón de unidades formadoras de colonias (ufc) dentro de un período relativamente corto a temperatura ambiente, mientras que calentar la comida inmediatamente previo a la consumición puede reducir considerablemente los niveles de patógenos o sus toxinas (botulismo) $)^{11}$.

Como resultado, una microbiología predictora puede ser una herramienta útil en ejecutar el análisis de la exposición. Otros factores a ser considerados en la medición de la exposición incluyen pautas de consumo (el tamaño de la porción y la frecuencia de consumo) lo cual esta típicamente relacionado a trasfondos socioeconómicos y culturales, étnicos, estacionalidad, diferentes edades del consumidor (población demográfica), diferencias regionales, preferencias y conductas del consumidor, y el rol de los controles alimentarios como una fuente de contaminación ${ }^{1}$.

\section{Caracterización del riesgo}

Junto con la información cualitativa-cuantitativa de la caracterización del peligro y el análisis de la exposición, la determinación del riesgo provee una estimación del mismo para una población dada. Esta estimación puede ser medida por comparación con datos epidemiológicos independientes que relacionan la exposición a un alimento potencialmente peligroso y la prevalencia de enfermedad (ETA). El grado de confianza de la estimación final del 
riesgo dependerá de la variabilidad, incertidumbre y suposiciones identificadas en los pasos previos de ARM. La diferenciación de la incertidumbre y la variabilidad es importante en la selección subsecuente de opciones del manejo de los riesgos. La incertidumbre está asociada con los datos propiamente dichos e incluye aquellas que puedan surgir en la evaluación y extrapolación de la información obtenida de estudios epidemiológicos, microbiológicos y en animales de laboratorio. La incertidumbre se eleva cuando se hacen intentos para usar estos datos concernientes a determinados fenómenos obtenidos bajo ciertas condiciones, extrapolándolas para hacer estimaciones de predicciones sobre el fenómeno de ocurrencia bajo otras circunstancias para los cuales los datos no eran comparables ${ }^{12}$.

Un componente importante de la caracterización del riesgo son los análisis sensitivos. Estos sirven para determinar los parámetros que contribuyen mayormente a la total incertidumbre en el análisis del riesgo resultante. Un tipo común de análisis de sensibilidad es el usado por el escenario “ ¿qué pasaría si...?”. Usando esta metodología el asesor de riesgo puede examinar el impacto relativo de los cambios en diferentes parámetros en los modelos. Por ejemplo, uno podría encontrar en el modelo conceptual que un cambio en la prevalencia de patógenos en la granja tiene un efecto dramático en el riesgo. En este caso, los resultados de los análisis de sensibilidad indicarían que el mayor monto de recursos debería ser enfocado para reducir la prevalencia del patógeno en la granja. Los análisis de sensibilidad pueden también ser usados para identificar la brecha de datos cruciales. Estos parámetros conducen las estimaciones de riesgo, para lo cual hay muy pocos datos disponibles. El resultado final de la caracterización de riesgos es la estimación o predicción de enfermedades asociadas con un microorganismo particular, dada la incertidumbre y variabilidad inherente a la suposición y datos. El análisis de riesgo, tiene implicancias sobre las medidas de control ${ }^{7}$.

\section{Cómo son usados los resultados del análisis del riesgo}

Usualmente se trata de empezar por caracterizar los modelos estimativos de la "línea de base", comparando la predicción numérica de enfermedades con estimaciones basadas en estudios epidemiológicos. Ello es posible para examinar el impacto de diferentes conjeturas usadas en el modelo. Una importante característica de ECRM es la habilidad de examinar el impacto de intervenciones en distintos puntos a lo largo del proceso granja-mesa o la reducción de infecciones de origen alimentario y para identificar sobre cuáles puntos el impacto es más grande. La presentación de resultados de esta manera, facilita la estimación total de incertidumbre. Definir claramente los límites de confianza también ayuda a remarcar el hecho que los resultados son simplemente las mejores estimaciones, pero no absolutas ${ }^{3}$.

El principal resultado de un ECRM es la predicción de un número de casos, la gravedad de la enfermedad o ambos. El riesgo está basado sobre una base de datos o como estimaciones poblacionales. Esta última es útil cuando se enfoca un grupo de interés específico, tal como niños, ancianos o individuos inmuno-comprometidos. Las predicciones del ECRM son más frecuentemente dadas como distribuciones, en cuyo caso la mayor estimación está casualmente asociada a la distribución promedio o mediana. Las distribuciones demuestran un rango de resultados asociados con enfermedades de origen alimentario dentro de los límites de confianza superior e inferior (típicamente reportado con el percentil 5 y 95 de intervalo de confianza) para la estimación total sobre la incertidumbre y variabilidad ${ }^{14}$.

\section{Fase de gerenciamiento}

El gerenciamiento del análisis de riesgo está conformado por dos componentes:

\section{Estructura administrativa de las decisiones de riesgo (politicas)}

La gerencia del riesgo es el proceso de tomar decisiones sobre riesgos informados por el resultado del ARM calculado, sea éste aceptable o inaceptable, dado el consecuente nivel de incertidumbre. En el caso de riesgo inaceptable, la gerencia de riesgo está más probablemente involucrada en el desarrollo de certezas para reducir la exposición, y puede también incluir estrategias para la investigación o la recolección de datos adicionales para reducir la incertidumbre del riesgo estimado. En el caso de riesgo aceptable, la estrategia de gerencia de riesgo puede involucrar esfuerzos para asegurar que la exposición permanezca por debajo de cierto nivel ${ }^{4}$.

\section{La estrategia para la comunicación de riesgo}

Es un proceso de intercambio interactivo de información y opinión entre individuos, grupos e instituciones. Involucra múltiples mensajes sobre riesgos, que expresan preocupación, opinión o reacciones ante los mismos. Incluye la "traducción de la información" a la población general, potencialmente expuesta al riesgo determinado ${ }^{5}$.

\section{Clasificación de ARM según objeto de análisis}

El ARM puede ser caracterizado en, al menos, cuatro tipos específicos ${ }^{15}$ :

\section{Análisis de riesgo en elaboración de producto}

El propósito de conducir un análisis de riesgo en la elaboración de un producto es identificar los factores del 
proceso desde una planta industrial o desde la granja a la mesa, que son probables de afectar la salud pública relacionada a la ingesta de un patógeno por vía de una comida particular. Un ejemplo de este tipo de determinación es E. coli 0157:H7 aplicando ARM en hamburguesas. Un análisis de riesgo de este tipo está orientado con el modelo de crecimiento/decrecimiento de la bacteria a través del proceso granja-mesa. La fuerza del análisis de riesgo de este evento es tal que permite examinar los efectos predecibles de varias opciones de mitigación, incluidas aquellas relacionadas con la producción de animales, procesamiento de alimentos y la conducta del consumidor, sobre el riesgo de una ETA $^{15,16}$.

\section{Ranking de riesgo relativo}

El propósito primario de un riesgo relativo es identificar productos alimentarios que posean los riesgos mayores de enfermedad por un patógeno particular de origen alimentario. Un ejemplo de este tipo de medición es el análisis de riesgo para L. monocytogenes. Los ranking de riesgo relativo son particularmente útiles en instancias en que los datos epidemiológicos sugieren varios alimentos que pueden ser de preocupación para un particular patógeno. Los resultados del ranking de riesgo ayudan a enfocar los esfuerzos y recursos para una reducción de las $\mathrm{ETA}^{15}$.

\section{Determinación geográfica}

Estas son descritas para aclarar los caminos que son probables de llevar adelante en la exposición a un patógeno y los factores que bien limitan o bien permiten que el riesgo ocurra. Ellos son efectivos en identificar el riesgo de introducción de agentes de ETA por alimentos de origen animal o sus productos, y mitigaciones para reducir la exposición del consumidor a patógenos de origen alimentario ${ }^{18}$.

\section{Determinación riesgo-riesgo}

Inspecciona el intercambio de un riesgo por otro en situaciones en las cuales reducen el riesgo a un peligro y probablemente incrementa el riesgo de otro. Un ejemplo de este tipo de medición es el análisis del impacto en la salud pública tratando el agua de consumo o agua usada en procesos/manufacturas con un químico, digamos cloro, para eliminar un patógeno microbiano, Mycobacterium sp, por ejemplo. El riesgo de exposición al cloro, superando los niveles permitidos, podría ser esperado con un correlato inversamente proporcional al causado por la ingestión de $M$. tuberculosis ${ }^{19}$.

\section{Pronóstico de las necesidades de investigación (brechas de información)}

Identificar las brechas de la información es uno de los ejercicios más importantes en el análisis del riesgo en tanto provee información crítica que puede ser usada para priorizar y facilitar investigaciones futuras. Un buen reporte de análisis de riesgo es uno que clara y concisamente identifique los riesgos para ser tomados en cuenta o reportar futuras mediciones. Un ejemplo clásico de brechas de información comúnmente encontrada en $\mathrm{ARM}$, es la resultante de la escasa o casi inexistente información, relativamente accesible, la cual soporta la necesidad de realizar un modelo cuantitativo de la relación dosis/respuesta para muchos o la mayoría de patógenos implicados en $\mathrm{ETA}^{9}$.

\section{Algunos ejemplos del ECRM}

Análisis de riesgo de Escherichia coli O157:H7

Un ARM para E. coli O157:H7 fue llevado a cabo en E.U.A. por el Servicio de Inspección y Seguridad Alimentaria del Departamento de Agricultura, en colaboración con la Administración de Drogas y Alimentos, el Comité Asesor Nacional en Criterios Microbiológicos en Alimentos, la industria y las universidades. El estudio se inició como respuesta a la identificación de $E$. coli O157:H7 en ganado y carne molida, y a la luz de los serios impactos en la salud pública acusados por epidemias de esta bacteria propagadas a través de los alimentos. Dos de los principales propósitos de este trabajo fueron la evaluación comprensiva del riesgo de enfermedades por E. coli $\mathrm{O} 157: \mathrm{H} 7$ en la carne picada y la identificación de los puntos críticos en la cadena "de la granja a la mesa" donde las mitigaciones para reducir las enfermedades tienen más chances de ser efectivas. Fue un análisis de los riesgos de base, designado para reflejar todo el espectro de prácticas, desde la cría y producción de animales, la matanza, el procesado, el transporte, el almacenamiento, la preparación y el consumo. Muestras de carne fueron tomadas para examinar el riesgo de enfermedad causado por E. coli $\mathrm{O} 157: \mathrm{H} 7$ en porciones de carne picada; la exposición a otros productos, agua, contaminación cruzada y otras no fueron incluidas en el modelo de análisis de riesgo. Las estimaciones resultantes fueron concebidas para cubrir una variedad de modelos de análisis ulteriores, incluyendo riesgos de enfermedad, de hospitalización, de ocurrencia de síndrome hemolítico urémico y de muerte ${ }^{6}$.

En agosto de 1998, el Servicio de Inspección y Seguridad Alimentaria del Departamento de Agricultura anunció sus planes para desarrollar un estudio de análisis de riesgo centrado en la enfermedad en humanos por $E$. coli $\mathrm{O} 157: \mathrm{H} 7$ en la carne picada. La información científica disponible al primero de julio de 2001 fue compilada, analizada e integrada en el modelo de análisis de riesgo. La Academia Nacional de Ciencias fue contratada para armar un panel de expertos que condujese una "revisión de pares" (la verificación por parte de expertos en la materia de los resultados de un estudio). Como resultados y con- 
clusiones del ARM se predijo que menos de 0,007-0,018\% (dependiendo de la época del año) de los productos de carne picada cocida contenían E. coli O157:H7. La posibilidad mediana de enfermedad proveniente de la misma para la población estadounidense se predijo en aproximadamente 1 cada millón de porciones. Esta predicción era fuertemente dependiente de la época del año en la cual la carne picada es consumida (1/600.000 en la época junio-septiembre -verano boreal- contra 1/1.600.000 en octubre-mayo). Los niños bajo cinco años de edad tendrían un riesgo de enfermedad de alrededor de 2,5 veces más que el de la población general. Se identificó la necesidad de mayor investigación en las siguientes áreas: el efecto que el enfriado de la res tiene en la disminución (o aumento) del número de $E$. coli $\mathrm{O} 157: \mathrm{H7}$; la influencia de las inundaciones en la zona productiva, los microbios que compiten con $E$. coli y las condiciones ambientales sobre la densidad máxima de $E$. coli en la carne picada, y el almacenamiento en los locales de venta y referido a los consumidores, la cocción y los patrones de consumo de carne picada según la estación del año ${ }^{21}$.

De manera similar a lo que ocurre en el mercado de trabajo estadounidense con las estadísticas de empleo, las evaluaciones científicas y los modelos matemáticos desarrollados para el ARM proveyeron una evaluación del conocimiento científico en su rol de asistencia a la revisión de la efectividad de las políticas, programas y prácticas que se desarrollaban en ese momento, y permitieron identificar estrategias para reducir el impacto de $E$. coli O157:H7, presente en la carne picada, en la salud pública. Por ejemplo, el ARM fue utilizado para determinar el efecto de una serie de mitigaciones en los mataderos. También, los resultados proveyeron una base para determinar ulteriormente que $E$. coli $\mathrm{O} 157: \mathrm{H} 7$ tiene una posibilidad de ocurrencia razonable en la carne picada ${ }^{3}$.

\section{Análisis de riesgo para Listeria monocytogenes}

$\mathrm{El}$ análisis de riesgo para L. monocytogenes (LARM), fue realizado en E.U.A. por las agencias federales para reducir la listeriosis en $50 \%$ en el año 2005. El propósito de esta medición fue identificar cuáles alimentos debían recibir la mayor atención regulatoria en un esfuerzo para mejorar la salud pública. El punto final de la enfermedad usada en el ARM era la fatalidad del paciente. El número de casos de listeriosis estaba estimado a partir de muertes predecibles, usando un factor de corrección basado en información FoodNet (red de vigilancia activa de enfermedades transmitidas por los alimentos). El ARM provee análisis y modelos que estiman el nivel potencial de exposición de tres grupos de población basados en edad (perinatal, edad intermedia y ancianos) y la población total de E.U.A. para L. monocytogenes en comidas contaminadas para 23 categorías de alimentos, y relacionan esta exposición a las consecuencias en la salud pública. Los modelos de LARM proveyeron un promedio estimado de infección fatal de L. monocytogenes para una categoría de alimentos en particular ${ }^{22}$.

La investigación también proporcionó un número estimativo de infecciones letales por año en E.U.A. para cada categoría de alimentos, el "riesgo por año". Esto fue así pues el riesgo anual fue derivado del riesgo "por ciento", y había generalmente un grado más alto de incertidumbre asociado con este riesgo. Los resultados del estudio permitieron desarrollar diferentes enfoques para controlar la listeriosis basados en el riesgo relativo y características de comidas específicas (Tabla 1). Aunque el propósito de LARM no revisó los caminos de las manufacturas de alimentos individuales, los modelos observados pueden ser usados para estimar la probabilidad de impacto de las estrategias de control cambiando uno o más parámetros de entrada y midiendo el cambio en los modelos de salida. Las evaluaciones de los modelos científicos y matemáticos desarrollados por LARM proveyeron una

Tabla 1. Pronóstico de la mediana para casos de listeriosis en el total de la población de E.U.A. asociados a la ingesta de alimentos listos para el consumo, año 2005

\begin{tabular}{|lc|}
\hline Alimento implicado & Riesgo por porción \\
\hline Fiambres & $7,7 \times 10^{-8}$ \\
\hline Salchichas no recalentadas & $6,5 \times 10^{-8}$ \\
\hline Paté y otras pastas de carne & $3,2 \times 10^{-8}$ \\
\hline Leche fluida no pasteurizada & $7,1 \times 10^{-9}$ \\
\hline Pescados y mariscos ahumados & $6.2 \times 10^{-9}$ \\
\hline Crustáceos cocidos "listos para comer" & $5,1 \times 10^{-9}$ \\
\hline Lácteos de alto contenido graso & $2,7 \times 10^{-9}$ \\
\hline Quesos blandos sin madurar & $1,8 \times 10^{-9}$ \\
\hline Leche fluida pasteurizada & $1,0 \times 10^{-9}$ \\
\hline Quesos blandos frescos & $1,7 \times 10^{-10}$ \\
\hline Salchichas recalentadas & $6,3 \times 10^{-11}$ \\
\hline Pescado conservado & $2,3 \times 10^{-11}$ \\
\hline Pescado y mariscos crudos & $2,0 \times 10^{-11}$ \\
\hline Frutas & $1,9 \times 10^{-11}$ \\
\hline Salchichas fermentadas secas & $1,7 \times 10^{-11}$ \\
\hline Quesos semi-blandos & $6,5 \times 10^{-12}$ \\
\hline Quesos blandos maduros & $5,1 \times 10^{-12}$ \\
\hline Vegetales & $2,8 \times 10^{-12}$ \\
\hline Ensaladas de casas de comida & $5,0 \times 10^{-13}$ \\
\hline Helados y otros lácteos congelados & $4,9 \times 10^{-14}$ \\
\hline Quesos procesados & $4,2 \times 10^{-14}$ \\
\hline Productos de leche cultivada & $3,2 \times 10^{-14}$ \\
\hline Quesos duros & $4,5 \times 10^{-15}$ \\
\hline
\end{tabular}


medición sistemática de conocimiento científico necesario para asistir no sólo la efectividad de las revisiones de las políticas corrientes, programas y prácticas, sino también para identificar nuevas estrategias y así minimizar el impacto de L. monocytogenes de origen alimentario en la salud pública ${ }^{23}$.

\section{Otras evaluaciones de riesgo en la Seguridad Alimentaria}

Es imperioso el desarrollo y validación de modelos matemáticos para la predicción de vida comercial de productos cárnicos. En una investigación se elaboraron modelos predictores de crecimiento de L. mesenteroides subsp mesenteroides a partir de medidas de absorbancia en Bioscreen $\mathrm{C}$ en función del efecto combinado de las variables temperatura $\left(10,5-24,5^{\circ} \mathrm{C}\right)$, nivel de $\mathrm{pH}(5,5$ $7,5)$, concentración de cloruro sódico $(0,25-6,25 \%)$ y de nitrito sódico (0-200 ppm) bajo condiciones de aerobiosis y anaerobiosis en caldo MRS. Los datos de crecimiento de $L$. mesenteroides obtenidos a partir de medidas de absorbancia fueron ajustados al modelo primario de Baranyi y Roberts, el cual proporcionó los principales parámetros cinéticos (tasa máxima específica de crecimiento, fase de adaptación y densidad máxima de población). La temperatura fue el factor que ejerció un mayor efecto sobre la tasa máxima específica de crecimiento. Para la fase de adaptación fueron determinantes la temperatura y concentración de cloruro sódico. Por último, la concentración de cloruro sódico y nitritos, y en menor grado el nivel de $\mathrm{pH}$, fueron los factores de mayor influencia sobre la densidad máxima de población. Se observaron diferencias significativas en el comportamiento de L. mesenteroides en condiciones de aerobiosis y anaerobiosis. De los tres tipos de modelos aplicados a los datos, según la validación matemática, en aerobiosis se observó que el modelo de Redes Neuronales (RN) individual fue el que realizó predicciones más exactas de los parámetros cinéticos tasa máxima específica de crecimiento (max) y densidad máxima de población (yEnd) de L. mesenteroides, mientras que para la fase de adaptación tanto el modelo de RN individual como el modelo de Respuesta en Superficie estimaron adecuadamente este parámetro. En anaerobiosis, el modelo de RN individual fue el que mejor estimó los parámetros, maxy y End de dicho microorganismo. En ambas atmósferas gaseosas, los valores observados para los índices Bf y Af fueron próximos a 1 , variando entre $0,95-1,23$ para el factor sesgo y entre 1,14 y 1,31 para el factor exactitud ${ }^{21}$.

Otro interesante aporte fue realizado por el empleo de fermentos lácticos en la fabricación de productos cárnicos. En este trabajo se ha realizado un estudio comparativo sobre un típico embutido crudo curado, el salchichón "tipo casero", que se elaboró con diferentes cultivos iniciadores y bio-conservadores. Para ello se fabricaron cinco lotes de embutidos de 42 piezas cada uno, dos de estos lotes se inocularon con cultivos comerciales, otros dos con bio-conservadores y otro, al que no se le adicionó nada, se consideró el lote control. Estos salchichones se maduraron en una cámara de maduración controlada durante 30 días. A lo largo de todo el proceso se realizaron distintos análisis químicos y físico-químicos (composición química bruta, evolución de $\mathrm{pH}$ y $\mathrm{A}_{\mathrm{w}}$, cantidad de cloruro sódico, nitritos y evolución de los índices de oxidación de la grasa). También se estudiaron los distintos grupos de microorganismos, tanto los involucrados en la maduración como los alterantes y patógenos, con el fin de valorar la posible acción bio-conservadora de los productos añadidos. Y finalmente, se realizó una evaluación sensorial para definir las características organolépticas de los distintos lotes de salchichones y poder identificar las posibles diferencias que originaban la adición de uno $\mathrm{u}$ otro tipo de cultivo láctico. En los resultados obtenidos no se aprecian diferencias significativas $(>0,05)$ en la mayoría de los parámetros químicos y físico-químico, a excepción del $\mathrm{pH}$ y el contenido de ácido láctico. Todos los embutidos analizados cumplieron la Norma de Calidad para salchichón de categoría extra. En cuanto a los parámetros microbiológicos, los salchichones inoculados con cultivos iniciadores comerciales mostraron recuentos de lactobacilos y micrococáceas significativamente más elevados $(\mathrm{p}<0,001)$ que el resto de lotes. Asimismo, estos lotes de embutido mostraron los menores recuentos de enterobacterias, coliformes y enterococos. Estos grupos microbianos también fueron significativamente más bajos $(p<0,001)$ en los embutidos inoculados con uno de los bio-conservadores (lote b). Con respecto a los atributos sensoriales no se observaron diferencias significativas $(p>0,005)$ entre los distintos lotes de salchichones. De estos resultados se puede deducir que, aunque los bioconservadores podrían utilizarse como un obstáculo más en la conservación de los embutidos crudos curados, no llegarían a reemplazar a los cultivos iniciadores en la dirección y orientación de la fermentación láctica ${ }^{24}$.

Otros autores profundizaron en los aspectos metodológicos de la ECRM en productos cárnicos cocidos mediante la aplicación de modelos matemáticos, haciendo especial hincapié en los fenómenos de contaminación cruzada como un factor clave en el riesgo alimentario, al igual que su impacto sobre la Gestión del Riesgo (GR). El estudio exhaustivo de los fenómenos de contaminación cruzada revela que la presión, humedad, y tiempo de contacto son importantes factores de riesgo. Entre las carencias detectadas se encuentra la falta de modelos matemáticos de contaminación cruzada para la ECRM. En este sentido, se realizó un estudio experimental de contaminación cruzada para Staphylococcus aureus y E. coli O157:H7, y se elaboran modelos matemáticos con el propósito de que puedan ser aplicados en la ECRM. Igualmente, se incluyó 
un modelo de contaminación cruzada en la Evaluación de Exposición dentro de un esquema de ECRM usando un diseño con escenarios para un caso estudio de L. monocytogenes en productos cárnicos cocidos. El concepto de Objetivo de Seguridad Alimentaria se empleó como criterio para valorar el riesgo de cada escenario de contaminación cruzada. Finalmente, se realiza un desarrollo formal de una ECRM para L. monocytogenes en productos cárnicos cocidos, aplicando un análisis de Monte-Carlo de dos dimensiones. A través de la aplicación de métodos de sensibilidad, se identificaron aquellos factores más relevantes en el riesgo de listeriosis al igual que las fuentes de incertidumbre más importantes en la estimación final del riesgo. Esta información es la base para el desarrollo de la etapa de GR. Con esta investigación se impulsó una nueva metodología, la ECRM como base para el establecimiento de políticas adecuadas de Gestión de Riesgo Microbiano en Alimentos ${ }^{25}$.

Uno entre otros varios estudios se desarrolló con el fin de establecer un modelo de crecimiento microbiano de $E$. coli enterohemorrágica en carne vacuna (hamburguesas) como parte de una evaluación cuantitativa de riesgos. Se seleccionaron artículos científicos que exponían de manera completa modelos predictores de crecimiento del patógeno en carne, en función de la temperatura y dos modelos predictores terciarios. A partir de éstos se generaron datos sobre el tiempo de latencia (ë) y tasa de crecimiento $(\mu)$ en un rango de temperaturas $\left(5^{\circ} \mathrm{Ca} 34^{\circ} \mathrm{C}\right)$ y $\mathrm{pH}(5,6-6,5)$, obteniéndose la relación lineal entre cada parámetro y temperatura. El modelo empleado consideró un amplio rango de temperaturas y $\mathrm{pH}$, respondiendo a las condiciones normales de almacenamiento bajo refrigeración de este tipo de productos. El modelo predictor microbiológico que mejores resultados generó es el de Gompertz, el cual, al incorporar como parámetro la concentración de bacterias que alcanzan la fase estacionaria de crecimiento, evita obtener valores extremadamente elevados y produce resultados biológicamente razonables ${ }^{26}$.

Un gran número de evaluaciones de riesgo alimentario han sido llevadas a cabo. La Tabla 2 muestra algunos ejemplos ${ }^{1}$.

\section{A modo de second-look}

En el caso de alimentos, y en particular de aquellos que serán consumidos en estado fresco (como es común en hortalizas y frutas), la decisión de adquirir el producto tiene que ver con la seguridad, tranquilidad o confianza de que al hacerlo no se afectará la salud del consumidor. Al de inocuidad está asociado el concepto de riesgo ${ }^{5}$. Riesgo es la contingencia o probabilidad de que ocurra un daño. En el caso que nos interesa, el daño está vinculado con el perjuicio que pueda ocurrirle al consumidor (en su salud o aún en su vida). La inocuidad se define como la certeza de que un alimento o ingrediente utilizado en cantidad o de una manera acostumbrada y razonable no será causa de una lesión o un daño en el consumidor ${ }^{9}$.

En relación con los productos frescos, se suele clasificar estos riesgos en los siguientes.

- Riesgos biológicos, transmitidos por microorganismos.

- Riesgos químicos, provocados por contaminantes naturales o añadidos.

- Riesgos físicos, por la intrusión de materiales extraños.

A modo de ejemplo, una guía para la reducción del riesgo microbiano con énfasis en frutas y verduras frescas debería incluir los siguientes principios ${ }^{11}$ :

Principio $\mathrm{N}^{\circ} 1$. Es preferible prevenir la contaminación microbiana de frutas y vegetales.

Principio $\mathrm{N}^{\circ}$ 2. Para reducir al mínimo el riesgo microbiano, los agricultores, empacadores y transportistas deben usar buenas prácticas agrícolas y manufactureras.

Principio $\mathrm{N}^{\circ} 3$. Las frutas y vegetales frescos pueden entrar en contacto con contaminantes microbianos en cualquier punto de su trayectoria de la granja a la mesa. La mayoría de los microorganismos patógenos en estos alimentos provienen de las heces de los seres humanos o de los animales.

Principio $\mathrm{N}^{\circ}$. Cuando el agua entra en contacto con las frutas y vegetales frescos, la calidad y procedencia de la misma determina la posibilidad de contaminación por esta fuente.

Principio $\mathrm{N}^{\circ}$ 5. En relación con los riesgos por el uso de estiércol animal, éstos se derivan por la presencia de microorganismos patógenos en los intestinos de los animales. Entre otros, Salmonella, Cryptosporidium, Escherichia coli, Enterococus. Todos ellos afectan la salud humana.

La recomendación principal para disminuir los riesgos citados es la transformación de los desechos orgánicos en abono. El proceso es conducido por bacterias y hongos que al fermentar el material orgánico, generan mucho calor, reduciendo o eliminando los riesgos biológicos.

Principio $\mathrm{N}^{\circ}$ 6. La higiene y prácticas sanitarias de los trabajadores durante la producción, recolección, selección, empaque y transporte juegan un papel esencial en reducir lo más posible el riesgo de contaminación microbiana de frutas y vegetales frescos.

Principio $\mathrm{N}^{\circ} 7$. Hay que cumplir con todos los reglamentos de los gobiernos locales y estatales y las correspondientes leyes sobre buenas prácticas agrícolas.

Principio $\mathrm{N}^{\circ} 8$. Hay que investigar nuevas metodologías como la determinación no destructiva de parámetros de calidad de frutas y hortalizas mediante espectroscopia de reflectancia en el infrarrojo cercano.

Principio $\mathrm{N}^{\circ}$ 9. Para que el programa de seguridad alimentaria de buenos resultados es importante una 


\section{Tabla 2. Resumen de las más recientes Evaluaciones de Riesgo Microbianos para la Seguridad Alimentaria}

\section{Patógeno/producto de interés \\ Bacillus cereus en leche pasteu- rizada}

Campylobacter spp en pollo a la parrilla y Vibrio spp en mariscos

Campylobacter spp en pollo

Campylobacter spp en pollo (fluoroquinolona resistentes )

Centro de Medicina Veterinaria, FDA, E.U.A. (2001)

Escherichia coli en carne picada Health Canada (1998)

Escherichia coli en steak tartare (preparación similar a una hamburguesa, pero cruda)

Listeria monocytogenes en fiambres

Departamento de Agricultura (E.U.A.), (2003)

Listeria monocytogenes en quesos de pasta blanda

Listeria monocytogenes en algunas categorías de comidas listas para consumir

Instituto Nacional de Salud Pública y Medio Ambiente, Países Bajos (2001)

$$
\text { (E.U.A.), (2003) }
$$

Laboratorio de Epidemiología y Escuela de Veterinaria Francia. (1998)

Departamento de Salud y Servicios Humanos, Departamento de Agricultura (E.U.A.), (2003)

Salmonella enteritidis en huevo líquido pasteurizado

Centro para la Seguridad Alimentaria y Nutrición, Departamento de Agricultura (E.U.A.), (1997)

Salmonella enteritidis en huevo con cáscara

Salmonella Typhimurium DT104 en salchichas de cerdo secas

\section{(E.U.A.), (2005)}

Concejo Danés de la Carne y el Tocino, Comité Nacional para la Producción del Cerdo, e Instituto Danés de Investigación Cárnica. (2002)

Vibrio parahaemolyticus en moluscos crudos

\section{Resumen de resultados}

El almacenamiento a una temperatura de $7^{\circ} \mathrm{C}$ o menos, por no más de 7 días (condiciones de almacenaje de leche pasteurizada en Países Bajos), predice que el inoculo de $B$. cereus presente en la leche a la fecha de vencimiento no exceda los $10^{5} \mathrm{ufc} / \mathrm{mL}$

Acciones como el lavado a fondo y la preparación segura de mariscos fueron identificadas como las mitigaciones clave para reducir los riesgos de enfermedad causada por vibrios en estos alimentos Los resultados del análisis de riesgo, basados en datos de los programas de vigilancia daneses, sugieren que la incidencia de campilobacteriosis proveniente del consumo de pollo se reduciría unas 30 veces tomando una de estas medidas: Reducir el número de Campylobacter a la mitad en el esqueleto de los pollos. Reducir la prevalencia de Campylobacter en las bandadas unas 30 veces. Mejorar la higiene de las cocinas unas 30 veces. Se predice que el grupo etáreo de 18-29 años presenta el mayor riesgo de campilobacteriosis proveniente del pollo

Esta evaluación proveyó estimaciones cuantitativas para el riesgo a la salud humana que plantea este patógeno. Las estimaciones fueron extraídas del número de pacientes estadounidenses infectados que fueron tratados con fluoroquinolonas

El modelo predijo una probabilidad de 3,7 × 10-6 de contraer síndrome hemolítico urémico y de $1,9 \times 10^{-7}$ de fallecimiento, por porción, para la población más joven. Se predijo una reducción del $80 \%$ en la probabilidad promedio de enfermedad si se introducía una hipotética estrategia de mitigación, dirigida a reducir el crecimiento microbiano durante el almacenamiento para la venta a través de la reducción de la temperatura de almacenamiento

Este análisis de riesgo "de la granja a la mesa" arrojó resultados que sugieren que la prevalencia de hamburguesas crudas contaminadas por $E$. coli productora de toxinas shiga es baja en Países Bajos $(0,3 \%)$, pero la tasa de incidencia de enfermedades diarreicas es alta (8 cada 100.000 personas). Se predice que la intervención en la granja o durante la matanza del animal es más eficiente en la reducción de los riesgos aparejados por $E$. coli que la intervención del consumidor

Las superficies de contacto de la comida en las que se encontró Listeria spp. incrementan enormemente la posibilidad de encontrar productos listos para el consumo positivos en L. monocytogenes. Se predijo que intervenciones múltiples, que incluyen incrementar la inspección y sanidad de las superficies de contacto con los alimentos, el uso de inhibidores de crecimiento y la reformulación de los productos son más efectivas en reducir el riesgo de enfermedades asociadas que cualquier medida aislada

El número promedio de casos esperados de listeriosis por año en Francia se estimó en 57 para una subpoblación de alto riesgo y en 1 para una de bajo riesgo y buena salubridad. Reduciendo la contaminación ambiental de la leche y eliminando la mastitis bovina se reduce sustancialmente la incidencia esperada de listeriosis

Los resultados apoyaron los hallazgos de investigaciones epidemiológicas sobre que ciertos alimentos son más propensos a ser vehículos para listeria, y cuantificaron la magnitud de las diferencias en el riesgo de listeriosis en bases anuales y por porción para distintas comidas listas para consumo. Los modelos de exposición y escenarios de simulación identificaron cinco factores que afectan la exposición del consumidor: cantidades y frecuencia de consumo; niveles de Listeria sp en los alimentos; potencial de la comida para soportar el crecimiento de Listeria sp durante el almacenamiento refrigerado; temperatura de la refrigeración; duración de la refrigeración

Los resultados revelaron que una inadecuada temperatura de pasteurización y/o el almacenamiento de los huevos a temperaturas abusivas entre la granja y la planta industrial incidían en la posibilidad de resultar en productos peligrosos de huevo líquido. Se predice que la pasteurización a temperaturas adecuadas ofrece al consumidor protección tanto contra la alta incidencia de aves infectadas con S. enteriditis como contra las altas temperaturas en el almacenamiento de los huevos

El rápido enfriamiento de los huevos luego de la puesta y su pasteurización fueron predichos para ser efectivos como prevención de la salmonelosis proveniente de huevos

Los descubrimientos de este análisis predijeron que cuando la salmonela está presente en el cerdo crudo, en general lo está en bajos números, y que durante su procesado la salmonela presente se reduce a aproximadamente 2 ó 3 unidades por estudio. Las salchichas curadas en seco, producidas en Dinamarca o importadas, contendrían infrecuentemente $S$. Typhimurium DT104, y aún en los casos en que se encuentra, el número de células bacterianas se predijo como bajo (1 a 4 células por porción)

Se encontró que la temperatura del agua en el momento de la recolección era la principal influencia en los niveles iniciales de $V$. parahaemolyticus en ostras. Había una significativa reducción de las probabilidades de enfermedad cuando las ostras eran enfriadas inmediatamente luego de la recolección 
actuación responsable a todos los niveles (en la finca, las instalaciones de empaque, el centro de distribución y el transporte). Hay que contar con personal preparado y un eficaz control para asegurar que todos los elementos del programa funcionen correctamente y se pueda rastrear el origen del producto a través de sus diversos canales de distribución ${ }^{1,9}$.

\section{Conclusiones}

La contaminación bacteriana de los alimentos se puede producir en cualquier etapa de la cadena, desde la producción primaria hasta la preparación para su consumo final y de ahí la necesidad de analizar cada problemática con una visión integral del tipo "de la granja a la mesa". Dentro de estos esquemas, el análisis de riesgos es un proceso estructurado y sistemático mediante el cual se examinan los posibles efectos nocivos para la salud como consecuencia de un peligro presente en un alimento, o de una propiedad de éste, y se establecen opciones para mitigarlos. Realizar una evaluación de los peligros microbiológicos resulta complejo por el potencial de las poblaciones bacterianas para crecer o disminuir en función del microambiente generado desde la producción hasta el consumo, lo cual obliga además a considerar las incertidumbres y variabilidad propias de dicho proceso biológico $^{24}$.

Las evaluaciones cuantitativas de riesgos presentan grandes desafíos a la hora de modelar el crecimiento microbiano, debido a la gran cantidad de factores que intervienen en dicho proceso (naturaleza de la matriz alimentaria, temperatura, duración del almacenamiento y características intrínsecas de cada microorganismo en particular) y a las formas existentes de modelar el desarrollo microbiano. De esta forma, en un modelo de evaluación de riesgos se deben considerar tanto la variabilidad como la incertidumbre asociada a los parámetros que definen las ecuaciones de crecimiento microbiano. En este contexto, la incertidumbre representa la falta de conocimiento sobre el valor de un parámetro, la cual puede reducirse si se realizan mediciones posteriores. Por su parte, la variabilidad representa la verdadera heterogeneidad de la población como consecuencia de su naturaleza biológica, la cual es irreductible empleando mediciones adicionales. En este marco, la incertidumbre puede ser resultado de mediciones imprecisas o falta de conocimiento de los efectos o condiciones que no están consideradas en el modelo, mientras que la variabilidad puede ser el resultado de cambios en la temperatura, diferencias entre cepas de una misma bacteria u otras fuentes de variabilidad biológica ${ }^{8,11}$.

La evaluación del ARM es una herramienta para brindar una información heterogénea en un modelo de trabajo organizado y sistemático. Es un enfoque con base científica, su desarrollo no es meramente una tarea técnica, sino que requiere interpretar los resultados científicos y adaptar los diferentes tipos de datos generados por distintas fuentes para responder a una pregunta. Cuando incluso en la mayoría de los casos la pregunta no fue generada por los datos obtenidos requiere más que simples habilidades técnicas. Para asegurar la implementación de una evaluación de riesgo de alta calidad se necesita un impresionante conjunto de conocimientos, experiencia práctica, perspectiva y, lo más importante, planeamiento cuidadoso ${ }^{13}$.

Surge también una cuestión: ¿cómo podemos mejorar y fortalecer el proceso de ARM? Aquí van algunas sugerencias: Deberían optimarse los métodos para comunicar la falta de certeza en datos y modelos utilizados para desarrollar la evaluación de riesgos ya que éstos son, como mínimo, escasos, y generalmente ignorados por aquellos responsables de la toma de decisiones. Es crítico tener un sistema que provea el tiempo y la experiencia para asegurar un análisis de alta calidad y balanceado. Cuando el tiempo u otros recursos son escasos, la calidad del análisis de riesgo tiene buenas chances de verse negativamente afectada. Cabe aclarar que detectar estos problemas resulta difícil sin un continuo ajuste de los métodos utilizados, realizado por expertos ${ }^{19}$.

Hay muchos tipos de ETA y más allá del entusiasmo por la evaluación de riesgos resulta imposible hacer un ARM para cada combinación patógeno/alimento existente. La priorización es fundamental. Hay que continuar también en la mejora de la eficiencia en el uso de recursos, incluyendo personal, experiencia, tiempo, finanzas, la oportunidad de colaboración y sinergias. Los analistas de riesgo son el recurso más importante para desarrollar un buen ARM. Los esfuerzos educativos, incluyendo los intentos por despertar el interés de los estudiantes en etapas tempranas de sus carreras, el desarrollo de programas y títulos universitarios en análisis de riesgo, y programas de educación continua para apuntalar el desarrollo profesional son necesarios para expandir el universo de analistas de riesgo bien calificados ${ }^{25}$.

Más allá de estas sugerencias, el ARM ha sido refinado a un punto donde es ampliamente reconocido como una útil herramienta para la toma de decisiones en ausencia de datos completos o confiables. Para que esta herramienta sea usada de forma más activa y eficiente, se debe tener en cuenta la cuidadosa planificación durante todo el proceso. Los resultados de un ARM no deben ser usados como la única base de la política o las decisiones regulatorias ${ }^{10}$, sino que deben ser llamados a complementar otra información, como datos epidemiológicos y de vigilancia, consideraciones financieras y la opinión y experiencia del consumidor a fin de permitir la toma de decisiones con mayor solidez. Utilizados de esta manera, los ARM proveen un válido recurso para recortar el impacto de 
infecciones transmitidas por los alimentos, y, finalmente, proteger la salud pública. El análisis de riesgos constituye un elemento clave del proceso de toma de decisiones contemporáneo, tanto para el gobierno como para la industria. Si bien no podemos saber qué nos depara el futuro, a la luz de la incertidumbre es necesario que tomemos decisiones informadas, realistas y fundamentadas.

Agradecimientos: Los autores agradecen al Prof. Nigel J. Fleischer, Overlook Hospital Summi, New Jersey, E.U.A. por la lectura crítica del manuscrito.

\section{Resumen}

Las metas básicas del análisis de riesgo incluyen las siguientes: identificar las situaciones potencialmente peligrosas, aplicar los métodos apropiados para estimar la probabilidad que un peligro ocurra, y en la incertidumbre en esa estimación, proporcionar las soluciones alternativas para reducir el riesgo, estimar la eficacia de esas soluciones, proporcionar la información sobre las que se base una decisión de la gestión de riesgos, y estimar la incertidumbre asociada a la evaluación. El análisis de riesgos constituye un sistema de referencia coherente para reunir y analizar indicios sobre los factores de riesgo, y también para presentar los resultados de modo inteligible y obrar después eficazmente. Pero los progresos realizados no pueden excusar la arrogancia. La presente revisión no pretende sentar cátedra sobre el análisis de riesgo microbiano, más bien está pensado como una etapa más de un proceso necesario y permanente, como un jalón que describe el estado de cosas en 2010.

\section{Referencias}

1.- Forsythe S J. The Microbiology of Safe Food. $2^{\text {nd }}$ ed; Hardcover,Wiley-Blackwell 2010; p. 38-66.

2.- Waschmuth K, Kause J, Maczka C, Ebel E, Schlosser E, Anderson S. Microbial risk assessment as a tool for guiding food safety. New York, Marcel Dekker 2003; p. 79-102.

3.- Bower J. Statistical Methods for Food Science: Introductory procedures for the food practitioner. Hardcover, Wiley-Blackwell 2009; p. 228-44.

4.- Anderson E L, Hattis D. Uncertainty and variability. Risk Analysis 1199; 19: 47-9.

5.- Tucker G. Food Biodeterioration and Preservation. Hardcover, Wiley-Blackwell; 2007; p. 184-95.

6.- Rodricks J V. Calculated risk: the toxicity and human health risks of chemicals in our environment. Cambridge, UK, Cambridge University Press 1992; p. 256-78.

7.- Hofer, E, Kloos M, Krzykacz-Hausmann B, Peschke J, Woltereck M. An approximate epistemic uncertainty analysis approach in the presence of epistemic and aleatory uncertainties. Reliabil Engin Syst Safe 2002; 77: 3, 229-38.

8.- Forsythe S J. The Microbiological Risk Assessment of Food. Hardcover, WileyBlackwell 2002; p. 166-92.

9.- Vose D. Risk analysis: a quantitative guide.
Chichester, UK, John Wiley \& Sons 2000; p. 118-59.

10.- Clemen R T, Winkler R L. Combining probability distributions from expert in risk analysis. Risk Analysis 1999; 29: 187-203.

11.- Schroeder C M, Jensen E, Miliotis M D, Dennis S B, Morgan M K. Microbial risk assessment. In: Foodborne Diseases, Shabbir Simjee ed, , Hampshire, UK, Humana Press Inc; 2007; p. 435-56.

12.- Kaplan S. The words of risk analysis. Risk Analysis 1997; 17: 407-17.

13.- Dennis S B, Miliotis M D, Buchanan R L. Hazard characterization/dose-response assessment: In: Microbial risk assessment in food processing. Bronwn, M, Striger, M., eds., Cambridge, UK, Woodhead 2002; p. 83-6.

14.- Apeland S, Aven T. Risk based maintainance optimization: foundational issues. Reliabil Engin Syst Safe 2000; 67: 285-92.

15.- Klapwijk P M, Jouve J L, Stringer M F. Microbiological risk assessment in Europe: the next decade. Int J Food Microbiol 2000; 58: 223-30.

16.- Davies K W. Design of experiments for predictive microbial modeling. J Industr Microbiol 1993; 12: 296-300.

17.- Whiting R C, Bagi L K. Modeling the lag phase of Listeria monocytogenes. Int J Food Microbiol 2002; 73: 291-5.

18.- Side C. Food product development: based on experience. Hardcover, Wiley-Blackwell; 2002; p. $272-86$

19.- Clemen R T, Reilly T. Making hard decisions with decision tools. California, U.S.A, Duxbury Thomson Learning, Pacific Grove; 2001; p. 136-62.

20.- Hills B P, Wright K M. A new model for bacterial growth in heterogeneous systems. J Theor Biol 1994; 168: 31-41.

21.- Tamine A D. Milk Processing and Quality Management. Hardcover, Wiley-Blackwell 2009; p. 59-110.

22.- Mosleh A, Bier V M, Apostolakis G. A critique of current practice for the use of expert opinions in probabilistics risk assessment. Reliabil Engin Syst Safe 1988; 20: 63-85.

23.- McMeekin T A, Olley JN, Ross T, Ratkowsky D A. Predictive microbiology: theory and application. Research Studies Press, Exeter; 1993; p. 229-43.

24.- Evans J R, Olson D L. Introduction to simulation and risk analysis. New Jersey, U.S.A., Prentice Hall; 2002, p. 63-85.

25.- Haas C N, Rose J B, Gerba C P. Quantitative microbial risk assessment. New York, U.S.A., John Wiley and Sons 1999; p. 307-28.

26.- Tamplin M L, Paolib G, Marmera B S, Pillips J. Models of the behavior of Escherichia coli $\mathrm{O} 157: \mathrm{H} 7$ in raw sterile ground beef stored at 5 to $46{ }^{\circ} \mathrm{C}$. Int. J. Food Microbiol 2005; 100 : $335-44$. 